\title{
Novel Queueing Model for Multimedia Over Downlink in 3.5G Wireless Network
}

\author{
Khalid Al-Begain, Alexander N. Dudin, and Vilena V. Mushko
}

\begin{abstract}
In this paper, a model for multimedia transmission over downlink shared channel in $3.5 \mathrm{G}$ wireless network is presented. The multimedia stream consists of multiple substreams that are aggregated into one real-time and one nonreal-time flows. Correlation with each flow and between flows is assumed. Additionally, we propose a combined time-space priority buffer management scheme to optimise quality of service requirements for each flow. The problem is formulated in terms of a queue with two priority classes, one of which has time priority while the another has space priority. The input is described by the Batch Marked Markovian Arrival Process (BMMAP). Service time distributions are of $P H$ (phase) type dependent on the class of a customer. The buffer is finite, but the customers of a class having higher priority for taking into the service from a buffer (time priority) can occupy only a part of this buffer. Queueing system's behavior is described in terms of multi-dimensional continuous time skip-free to the left Markov chain. It allows to exploit an effective algorithm for calculation of the stationary distribution of the queueing system. Loss probability for customers of both classes is calculated. Waiting time distribution for priority customers is calculated.
\end{abstract}

Index terms: 3.5G wireless networks, multimedia traffic, stochastic models, performance modelling

\section{INTRODUCTION}

While Third Generation (3G) wireless systems are being intensively deployed worldwide, new proposals for enhanced data rates and quality of service provision are being standardised. One of the most promising enhancements to the widely deployed Universal Mobile Telecommunication System (UMTS) which is based on Wideband Code Division Multiple Access (W-CDMA) is called High Speed Downlink Packet Access (HSDPA) [1] which is also referred to as $3.5 \mathrm{G}$. HSDPA aims at providing data rates around $2-10 \mathrm{Mbps}$ on the downlink to mobile users mainly for multimedia services such as real-time and streaming video in packet-switched common channel.

On one hand, modelling of multimedia traffic over shared channel is rather complicated. Therefore, most of the studies typically investigate the problem using packet-level simulation [4] or as data flows which can be real-time (voice or video) or non-real-time (www browsing, e-mail, ftp, or data access).

Manuscript received January 01, 2006 and revised May 04, 2006.

$\mathrm{K}$. Al-Begain is with the Mobile Computing, Communications and Networking RG, School of Computing University of Glamorgan, Pontypridd, Wales, UK, (e-mail: kbegain@glam.ac.uk).

A. N. Dudin and V. V. Mushko are with the Laboratory of Applied Probabilistic Analysis, Department of Applied Mathematics and Computer Science, Belarusian State University, Minsk, Belarus (e-mail: dudin@bsu.by; vmushko@tut.by)
Other works considered performance study of the HSDPA system taking into account system details rather than the multimedia traffic characteristics [15]. Attempts to capture some characteristics of the multimedia traffic analytically were made by several authors such as using self-similar traffic [6], [10], Generalised Exponential (GE) Distribution [3], or Batch Markov Arrival Process [5], [7], [13]. A common weakness in all previous models is that even if they capture correlation which an important feature of the multimedia traffic ( $G E$ does not even capture correlation), they consider the two flows independent of each other. However, even the single multimedia stream is composed of two types of traffic; real-time (voice and/or video) and non-real-time (data) traffic flows. These flows cannot be considered as independent flows. This implies the necessity of considering two types of correlation; intra-flow and inter-flow correlation within the same multimedia stream.

On the other hand, providing Quality of Service (QoS) for multimedia traffic requires differentiated consideration of the different flows within the single multimedia stream. The realtime traffic flow requires low delay and low jitter but can tolerate some packet loss while the data component (non-realtime) is very sensitive to packet loss but can tolerate some delay or jitter. In [2], the authors presented a multi-service class buffer model that can provide time and space prioritization based on thresholds Both priorities, however, can only be given to the same service or flow. In addition, the multimedia traffic was modelled by the $G E$ distribution.

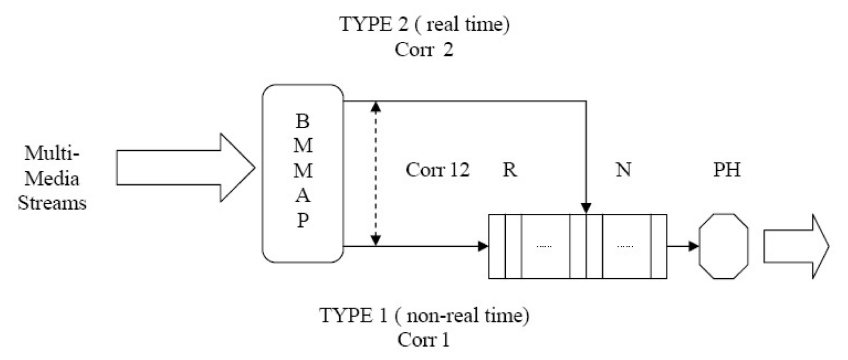

Fig. 1. A simplified block diagram of the proposed buffer architecture

The paper reports on initial results of a new line of research that will bring new dimension to the analytical modelling of multimedia traffic over wireless channels. We consider a multimedia stream that is composed of two types of traffic; real-time (voice and/or video) and non-real-time (data) traffic streams (See Figure 1). Unlike most recent studies, our models present two novel features that aim at providing necessary prioritization for QoS provisioning of multimedia services. 
First, we consider a novel queuing model for the buffer in which the two classes of traffic that compose the one multimedia stream enjoy different priority types that suits their QoS requirement; namely time priority for real-time traffic which a threshold to control the delay and jitter while we provide space priority for the data in order to minimize packet loss. In our project, we consider a number of distributions to model both the arrival process and the service process of the multimedia traffic. In this paper, we consider a novel application of the Batch Marked Markovian Arrival Process $(B M M A P)$ to represent the multimedia stream that consists of two classes of traffic. The main advantage of using $B M M A P$ is that it is capable of capturing both the intra-flow and interflow correlation.

This paper represents the report on the results of this project, and contains the formulation of the model, its analysis and the results of numerical experiments. It provides the solution and formulae for calculating packet loss probabilities for both classes and derivation of waiting time distribution for type-2 flow (real-time) will provide the tool to calculate the delay and jitter. This will then be the base for our optimization goal in which we use the threshold value to obtain desirable QoS for the multimedia traffic.

\section{MATHEMATICAL MODEL}

We have a single-server queue with a finite buffer of a capacity $R, R>0$. The input flow is described by the $B M M A P$ [9]. Customers arrival in the BMMAP is directed by the irreducible continuous time Markov chain $v_{t}, t \geq 0$, with a finite state space $\{0,1, \ldots, W\}$. Sojourn time of the chain $v_{t}, t \geq 0$, in the state $v$ has exponential distribution with a parameter $\lambda_{v}$. After this time expires, with probability $p_{0}\left(v, v^{\prime}\right)$ the chain jumps into the state $v^{\prime}$ without generation of customers and with probability $p_{k}^{(l)}\left(v, v^{\prime}\right)$ the chain jumps into the state $v^{\prime}$ and a batch consisting of $k$ customers of type $l$ (type- $l$ customers) is generated, $k \geq 1$. Here we will assume that only two types of customers are served in the system, so $l=1,2$. The introduced probabilities satisfy conditions:

$$
\begin{gathered}
p_{0}(v, v)=0, \\
\sum_{l=1}^{2} \sum_{k=1}^{\infty} \sum_{v^{\prime}=0}^{W} p_{k}^{(l)}\left(v, v^{\prime}\right)+\sum_{v^{\prime}=0}^{W} p_{0}\left(v, v^{\prime}\right)=1, v=\overline{0, W .}
\end{gathered}
$$

The parameters defining the BMMAP can be kept in the square matrices $D_{0}, D_{k}^{(1)}, D_{k}^{(2)}, k \geq 1$, of size $\bar{W}=W+1$ defined by their entries:

$$
\begin{gathered}
\left(D_{0}\right)_{v, v}=-\lambda_{v}, \\
\left(D_{0}\right)_{v, v^{\prime}}=\lambda_{v} p_{0}\left(v, v^{\prime}\right), \\
\left(D_{k}^{(l)}\right)_{v, v^{\prime}}=\lambda_{v} p_{k}^{(l)}\left(v, v^{\prime}\right), \\
v, v^{\prime}=\overline{0, W}, k \geq 1, l=1,2 .
\end{gathered}
$$

Denote

$$
\begin{gathered}
D(1)=D_{0}+\sum_{l=1}^{2} \sum_{k=1}^{\infty} D_{k}^{(l)}, \\
\hat{D}_{k}^{(l)}=\sum_{i=k}^{\infty} D_{i}^{(l)}, k \geq 1, l=1,2,
\end{gathered}
$$

$\boldsymbol{\theta}$ is the stationary probability vector of the states of the Markov chain $v_{t}, t \geq 0$.

Vector $\boldsymbol{\theta}$ is the unique solution to the system

$$
\boldsymbol{\theta} D(1)=\mathbf{0}, \boldsymbol{\theta e}=1 \text {. }
$$

Here and below $\mathbf{0}$ is the row vector of dimension which should be clear from context, $\mathbf{e}$ is the column vector of appropriate dimension consisting of all 1's.

Intensity $\lambda_{l}$ of type- $l$ customers arrival is calculated by

$$
\lambda_{l}=\boldsymbol{\theta} \sum_{k=1}^{\infty} k D_{k}^{(l)} \mathbf{e}, l=1,2
$$

Intensity $\lambda_{l}^{(b)}$ of batches of type- $l$ customers arrival is calculated by

$$
\lambda_{l}^{(b)}=\boldsymbol{\theta} \hat{D}_{1}^{(l)} \mathbf{e}, l=1,2 .
$$

Variation $v_{l}$ of inter-arrival times of batches of type- $l$ customers arrival is calculated by

$$
v_{l}=\frac{2 \boldsymbol{\theta}\left(-D_{0}-\hat{D}_{1}^{(\bar{l})}\right)^{-1} \mathbf{e}}{\lambda_{l}^{(b)}}-\left(\frac{1}{\lambda_{l}^{(b)}}\right)^{2}, \bar{l} \neq l, \bar{l}, l=1,2 .
$$

Squared coefficient of correlation $C_{c o r}^{(l)}$ of two successive intervals between type- $l$ customers arrival is computed by

$$
C_{c o r}^{(l)}=\left[\frac{\boldsymbol{\theta}\left(-D_{0}-\hat{D}_{1}^{(\bar{l})}\right)^{-1}}{\lambda_{l}^{(b)}} \hat{D}_{1}^{(l)}\left(-D_{0}-\hat{D}_{1}^{(\bar{l})}\right)^{-1} \mathbf{e}-\left(\frac{1}{\lambda_{l}^{(b)}}\right)^{2}\right] v_{l}^{-1},
$$

$$
\bar{l} \neq l, \bar{l}, l=1,2 .
$$

Coefficient of correlation $\quad C_{c o r}^{(l, \bar{l})}$ of two successive intervals between type- $l$ and type- $\bar{l}$ customers arrival (coefficient of cross correlation) is computed by

$$
\begin{gathered}
C_{c o r}^{(l, \bar{l})}=\left[\frac{\boldsymbol{\theta}\left(-D_{0}-\hat{D}_{1}^{(\bar{l})}\right)^{-1}}{\lambda_{l}^{(b)}} \hat{D}_{1}^{(l)}\left(-D_{0}-\hat{D}_{1}^{(l)}\right)^{-1} \mathbf{e}-\frac{1}{\lambda_{l}^{(b)} \lambda_{\bar{l}}^{(b)}}\right] \times \\
\times\left(\sqrt{v_{l}} \sqrt{v_{\bar{l}}}\right)^{-1}, \bar{l} \neq l, \bar{l}, l=1,2 .
\end{gathered}
$$

Type- 1 customers are accepted into the system if the buffer is not full. If the size of arriving batch exceeds the available space in a buffer we assume that the corresponding part of the batch is accepted into the buffer while the rest is lost. Such a discipline is called partial admission. Disciplines of complete admission or complete rejection where the whole batch is accepted or rejected correspondingly can be handled in a similar way.

Type- 2 customers have a priority with respect to type-1 customers. If at least one type- 2 customers presents in the 
system at the service completion epoch, then type-2 customer will get the service. Type-1 customers have a chance to get service only if no one type- 2 customer presents in the system. Interruption of the service is not allowed.

However, type- 2 customers have more restricted access into the system. No more then $N, 1 \leq N<R$, type- 2 customers can be accepted into the buffer. Discipline of partial admission is applied as well.

The service time of customers has $P H$ (phase) type distribution. It means the following. Service of type- $l$ customer is defined as a time until the continuous-time Markov chain $\eta_{t}^{(l)}, t \geq 0$, having the states $\left\{1, \ldots, M_{l}\right\}$ as the transient and state 0 as absorbing one reaches the absorbing state. The initial state of the chain is selected in a random way, according to the probability distribution defined by the rowvector $\left(\boldsymbol{\beta}_{l}, 0\right)$, where $\boldsymbol{\beta}_{l}$ is the stochastic row vector of dimension $M_{l}$. Transitions of the Markov chain $\eta_{t}^{(l)}, t \geq 0$, are described by the generator $\left(\begin{array}{cc}S^{(l)} & S_{0}^{(l)} \\ 0 & 0\end{array}\right)$, where the matrix $S^{(l)}$ is sub-generator and the column vector $S_{0}^{(l)}$ is defined by $S_{0}^{(l)}=-S^{(l)} \mathbf{e}$. The average service time is given by $\boldsymbol{\beta}_{l}\left(-S^{(l)}\right)^{-1} \mathbf{e}$. For more details about the $P H$ type distribution see [14].

Assumption that the arriving flow is the $B M M A P$ allows to catch correlation in the input process that is very important in modelling multimedia traffic. Assumption about $P H$ type service time distribution is some kind of trade-off between desire to consider the most general service process and possibility to have still analytically tractable Markov process as the model of the system behavior.

The proper selection of the buffer size $R$ and the threshold $N$, which restricts access of the priority customers, can allow effectively control the main performance measures of the system (delay and jitter for type-2 customers and loss probability for type-1 customers). As a first step in this direction, the problem of calculation of the stationary state distribution of the Markov chain, which will be described in the next section, should be solved. Mention that this stationary distribution exists always as the state space of the Markov chain is finite, the process $v_{t}, t \geq 0$, is assumed to be irreducible and the so called $\left(\boldsymbol{\beta}_{l}, S^{(l)}\right)$ representations of $P H$ are suggested to be irreducible.

\section{MARKovian Process of the System StateS}

Let

- $i_{t}^{(l)}$ be the number of type- $l$ customers presenting in a buffer at the epoch $t, l=1,2$;

- $\quad \xi_{t}$ be the type of the customer is service at epoch $t$;

- $v_{t}$ be the state of the directing process of the BMMAP at epoch $t$;
- $\eta_{t}^{\left(\xi_{t}\right)}$ be the state of the process which defines the service at the epoch $t$.

It is clear that the process

$$
\begin{gathered}
\zeta_{t}=\left\{i_{t}^{(2)}, i_{t}^{(1)}, \xi_{t}, v_{t}, \eta_{t}^{\left(\xi_{t}\right)}\right\}, t \geq 0, \\
i_{t}^{(2)}=\overline{0, N}, i_{t}^{(1)}=\overline{0, R-i_{t}^{(2)}}, v_{t}=\overline{0, W}, \eta_{t}^{(l)}=\overline{1, M_{l}}, l=1,2, \\
\xi_{t}= \begin{cases}l, & \text { if type }-l \text { customer is in the service, } l=1,2, \\
0, & \text { if the server is idle, }\end{cases}
\end{gathered}
$$

is the Markov chain. Denote the stationary state probabilities of this Markov chain by:

$$
\begin{gathered}
p(0,0,0, v)=\lim _{t \rightarrow \infty} P\left\{i_{t}^{(2)}=0, i_{t}^{(1)}=0, \xi_{t}=0, v_{t}=v\right\}, \\
p\left(i_{2}, i_{1}, r, v, \eta^{(r)}\right)= \\
\lim _{t \rightarrow \infty} P\left\{i_{t}^{(2)}=i_{2}, i_{t}^{(1)}=i_{1}, \xi_{t}=r, v_{t}=v, \eta_{t}^{(r)}=\eta^{(r)}\right\}, \\
i_{2}=\overline{0, N}, i_{1}=\overline{0, R-i_{2}}, v=\overline{0, W}, \eta^{(r)}=\overline{1, M_{r}}, r=1,2 .
\end{gathered}
$$

To simplify operation with the probabilities, we enumerate the states of the processes in the lexicographic order and introduce the vectors of stationary probabilities:

$$
\begin{gathered}
\mathbf{p}(0,0,0)=(p(0,0,0,0), p(0,0,0,1), \ldots, p(0,0,0, W)), \\
\mathbf{p}\left(i_{2}, i_{1}, r\right)=\left(p\left(i_{2}, i_{1}, r, 0,1\right), \ldots, p\left(i_{2}, i_{1}, r, 0, M_{r}\right),\right. \\
\left.p\left(i_{2}, i_{1}, r, 1,1\right), \ldots, p\left(i_{2}, i_{1}, r, 1, M_{r}\right), \ldots, p\left(i_{2}, i_{1}, r, W, M_{r}\right)\right), \\
r=1,2 .
\end{gathered}
$$

So, the row vector $\mathbf{p}(0,0,0)$ has dimension $\bar{W}$, the row vector $\mathbf{p}\left(i_{2}, i_{1}, r\right)$ has dimension $\bar{W} M_{r}, r=1,2$.

In what follows, we use the following denotations: $I$ is the identity matrix of dimension defined by the suffix, $\otimes$ and $\oplus$ are the symbols of Kronecker product and sum of the matrices correspondingly, $\delta_{i, j}$ is Kronecker delta:

$$
\delta_{i, j}= \begin{cases}1, & \text { if } i=j, \\ 0, & \text { if } i \neq j .\end{cases}
$$

Theorem 1. The vectors $\mathbf{p}(0,0,0), \mathbf{p}\left(i_{2}, i_{1}, r\right)$ satisfy the following system of equations:

$$
\begin{gathered}
\mathbf{0}=\mathbf{p}(0,0,0) D_{0}+\mathbf{p}(0,0,1)\left(I_{\bar{W}} \otimes S_{0}^{(1)}\right)+ \\
\mathbf{p}(0,0,2)\left(I_{\bar{W}} \otimes S_{0}^{(2)}\right),
\end{gathered}
$$

$$
\begin{gathered}
\mathbf{0}=\mathbf{p}\left(0, i_{1}, 1\right)\left(D_{0} \oplus S^{(1)}\right)+\mathbf{p}(0,0,0)\left(D_{i_{1}+1}^{(1)} \otimes \boldsymbol{\beta}_{1}\right)+ \\
\sum_{m=0}^{i_{1}-1} \mathbf{p}(0, m, 1)\left(D_{i_{1}-m}^{(1)} \otimes I_{M_{1}}\right)+\mathbf{p}\left(0, i_{1}+1,1\right)\left(I_{\bar{W}} \otimes S_{0}^{(1)} \boldsymbol{\beta}_{1}\right)+
\end{gathered}
$$




$$
\begin{aligned}
& \mathbf{p}\left(0, i_{1}+1,2\right)\left(I_{\bar{W}} \otimes S_{0}^{(2)} \boldsymbol{\beta}_{1}\right), i_{1}=\overline{0, R-1}, \\
& \mathbf{0}=\mathbf{p}\left(i_{2}, i_{1}, 1\right)\left(D_{0} \oplus S^{(1)}\right)+\sum_{m=0}^{i_{1}-1} \mathbf{p}\left(i_{2}, m, 1\right)\left(D_{i_{1}-m}^{(1)} \otimes I_{M_{1}}\right)+ \\
& \sum_{m=0}^{i_{2}-1} \mathbf{p}\left(m, i_{1}, 1\right)\left(D_{i_{2}-m}^{(2)} \otimes I_{M_{1}}\right), \\
& i_{1}=\overline{0, R-i_{2}-1}, i_{2}=\overline{1, N-1} \text {, } \\
& \mathbf{0}=\mathbf{p}\left(N, i_{1}, 1\right)\left(\left(D_{0}+\hat{D}_{1}^{(2)}\right) \oplus S^{(1)}\right)+ \\
& \sum_{m=1}^{i_{1}-1} \mathbf{p}(N, m, 1)\left(D_{i_{1}-m}^{(1)} \otimes I_{M_{1}}\right)+ \\
& \sum_{i_{2}=0}^{N-1} \mathbf{p}\left(i_{2}, i_{1}, 1\right)\left(\hat{D}_{N-i_{2}}^{(2)} \otimes I_{M_{1}}\right), i_{1}=\overline{0, R-N-1}, \\
& \mathbf{0}=\mathbf{p}\left(i_{2}, R-i_{2}, 1\right)\left(D(1) \oplus S^{(1)}\right)+ \\
& \sum_{m=0}^{R-i_{2}-1} \mathbf{p}\left(i_{2}, m, 1\right)\left(\hat{D}_{R-i_{2}-m}^{(1)} \otimes I_{M_{1}}\right)+ \\
& \sum_{l=0}^{i_{2}-1} \mathbf{p}\left(l, R-i_{2}, 1\right)\left(\hat{D}_{i_{2}-l}^{(2)} \otimes I_{M_{1}}\right), i_{2}=\overline{1, N} \\
& \mathbf{0}=\mathbf{p}(0, R, 1)\left(D(1) \oplus S^{(1)}\right)+\mathbf{p}(0,0,0)\left(\hat{D}_{R+1}^{(1)} \otimes \boldsymbol{\beta}_{1}\right)+ \\
& \sum_{m=0}^{R-1} \mathbf{p}(0, m, 1)\left(\hat{D}_{R-m}^{(1)} \otimes I_{M_{1}}\right),
\end{aligned}
$$$$
\mathbf{0}=\mathbf{p}\left(i_{2}, i_{1}, 2\right)\left(D_{0} \oplus S^{(2)}\right)+\sum_{m=0}^{i_{1}-1} \mathbf{p}\left(i_{2}, m, 2\right)\left(D_{i_{1}-m}^{(1)} \otimes I_{M_{2}}\right)+
$$$$
\mathbf{p}(0,0,0)\left(D_{i_{2}+1}^{(2)} \otimes \boldsymbol{\beta}_{2}\right) \delta_{i_{1}, 0}+\sum_{m=0}^{i_{2}-1} \mathbf{p}\left(m, i_{1}, 2\right)\left(D_{i_{2}-m}^{(2)} \otimes I_{M_{2}}\right)+
$$$$
\mathbf{p}\left(i_{2}+1, i_{1}, 1\right)\left(I_{\bar{W}} \otimes S_{0}^{(1)} \boldsymbol{\beta}_{2}\right)+\mathbf{p}\left(i_{2}+1, i_{1}, 2\right)\left(I_{\bar{W}} \otimes S_{0}^{(2)} \boldsymbol{\beta}_{2}\right),
$$$$
i_{2}=\overline{0, N-1}, i_{1}=\overline{0, R-i_{2}-1},
$$$$
\mathbf{0}=\mathbf{p}\left(N, i_{1}, 2\right)\left(\left(D_{0}+\hat{D}_{1}^{(2)}\right) \oplus S^{(2)}\right)+
$$$$
\sum_{m=1}^{i_{1}-1} \mathbf{p}(N, m, 2)\left(D_{i_{1}-m}^{(1)} \otimes I_{M_{2}}\right)+\sum_{i_{2}=0}^{N-1} \mathbf{p}\left(i_{2}, i_{1}, 2\right)\left(\hat{D}_{N-i_{2}}^{(2)} \otimes I_{M_{2}}\right)+
$$$$
\mathbf{p}(0,0,0)\left(\hat{D}_{N+1}^{(2)} \otimes \boldsymbol{\beta}_{2}\right) \delta_{i_{1}, 0}, i_{1}=\overline{0, R-N-1},
$$

$$
\begin{aligned}
& \mathbf{0}=\mathbf{p}\left(i_{2}, R-i_{2}, 2\right)\left(D(1) \oplus S^{(2)}\right)+ \\
& \sum_{m=0}^{R-i_{2}-1} \mathbf{p}\left(i_{2}, m, 2\right)\left(\hat{D}_{R-i_{2}-m}^{(1)} \otimes I_{M_{2}}\right)+
\end{aligned}
$$$$
\sum_{l=0}^{i_{2}-1} \mathbf{p}\left(l, R-i_{2}, 2\right)\left(\hat{D}_{i_{2}-l}^{(2)} \otimes I_{M_{2}}\right), i_{2}=\overline{0, N} .
$$

Proof. Proof of the theorem is implemented by means of analysis of intensity of transition of the process $\zeta_{t}, t \geq 0$.
Combine now the states corresponding to a fixed value of components $i_{2}$ and $r$ and introduce macro-vectors of stationary probabilities:

$$
\begin{gathered}
\tilde{\mathbf{p}}(0)=\mathbf{p}(0,0,0), \\
\mathbf{p}\left(i_{2}, r\right)=\left(\mathbf{p}\left(i_{2}, 0, r\right), \mathbf{p}\left(i_{2}, 1, r\right), \ldots, \mathbf{p}\left(i_{2}, R-i_{2}, r\right)\right), \\
i_{2}=\overline{0, N}, r=1,2 .
\end{gathered}
$$

Introduce the following notations of matrices:

- $\mathbf{B}_{i}^{(l, 2)}$ is the matrix of dimension $(R+1-i) \bar{W} M_{l} \times(R+2-i) \bar{W} M_{2}, \quad l=1,2$. For $i=\overline{1, N}$, this matrix represents the block diagonal matrix with diagonal blocks $I_{\bar{W}} \otimes S_{0}^{(l)} \boldsymbol{\beta}_{2}$ supplemented from the right side by the zero block column of dimension $(R+1-i) \bar{W} M_{l} \times \bar{W} M_{2}$;

- $\mathbf{B}_{i}^{(l, 1)}$ is the matrix of dimension $(R+1) \bar{W} M_{l} \times(R+2) \bar{W} M_{1}, l=1,2$. The matrix represents the block diagonal matrix with $R$ diagonal blocks $I_{\bar{W}} \otimes S_{0}^{(l)} \boldsymbol{\beta}_{1}$ supplemented from the right side by the zero block column of dimension $(R+1) \bar{W} M_{l} \times \bar{W} M_{1}$ and from above by the zero block row of dimension $\bar{W} M_{l} \times(R+1) \bar{W} M_{1}$;

- $\quad \boldsymbol{Z}^{(1)}$ has dimension $\bar{W} \times(R+1) \bar{W} M_{1}$ and is defined by

$$
\boldsymbol{Z}^{(1)}=\left(D_{1}^{(1)} \otimes \boldsymbol{\beta}_{1}, \ldots, D_{R}^{(1)} \otimes \boldsymbol{\beta}_{1}, \hat{D}_{R+1}^{(1)} \otimes \boldsymbol{\beta}_{1}\right) ;
$$

- $\quad \boldsymbol{T}^{(l)}$ has dimension $(R+1) \bar{W} M_{l} \times \bar{W}, \quad l=1,2$, and is defined as the block column having zero blocks except the first block which is equal to $I_{\bar{W}} \otimes S_{0}^{(l)}$;

- $\mathbf{D}_{i, j}^{(l)}$ is the matrix of dimension $(R-i+1) \bar{W} M_{l} \times(R-i-j+1) \bar{W} M_{l}, \quad j=\overline{1, N-i-1}$, $i=\overline{0, N-1}, \quad l=1,2$. It consists of the block diagonal matrix with the diagonal blocks $\left\{D_{j}^{(2)} \otimes I_{M_{l}}, \ldots, D_{j}^{(2)} \otimes I_{M_{l}}, \hat{D}_{j}^{(2)} \otimes I_{M_{l}}\right\} \quad$ supplemented from below by the zero matrix;

- $\quad \mathbf{D}_{i, N-i}^{(l)}$ has the analogous structure, but the blocks of the diagonal matrix are equal to $\hat{D}_{N-i}^{(2)} \otimes I_{M_{l}}, \quad l=1,2$;

- $\quad \boldsymbol{Z}_{i}^{(2)}$ has dimension $\bar{W} \times(R-i+2) \bar{W} M_{2}$ and is calculated by

$$
\begin{gathered}
\boldsymbol{\mathcal { Z }}_{i}^{(2)}=\left(D_{i}^{(2)} \otimes \boldsymbol{\beta}_{2}, O, \ldots, O\right), i=\overline{1, N}, \\
\boldsymbol{\mathcal { Z }}_{N+1}^{(2)}=\left(\hat{D}_{N+1}^{(2)} \otimes \boldsymbol{\beta}_{2}, O, \ldots, O\right)
\end{gathered}
$$

- $\quad \mathfrak{f}_{i}^{(l)}$ is the square matrix of dimension $(R-i+1) \bar{W} M_{l}$, $i=\overline{0, N-1}, l=1,2$, defined as follows: 


$$
\mathfrak{A}_{i}^{(l)}=\left(\begin{array}{cccccc}
D_{0} \oplus S^{(l)} & D_{1}^{(1)} \otimes I_{M_{l}} & D_{2}^{(1)} \otimes I_{M_{l}} & \cdots & D_{R-i-1}^{(1)} \otimes I_{M_{l}} & \hat{D}_{R-i}^{(1)} \otimes I_{M_{l}} \\
O & D_{0} \oplus S^{(l)} & D_{1}^{(1)} \otimes I_{M_{l}} & \cdots & D_{R-i-2}^{(1)} \otimes I_{M_{l}} & \hat{D}_{R-i-1}^{(1)} \otimes I_{M_{l}} \\
O & O & D_{0} \oplus S^{(l)} & \cdots & D_{R-i-3}^{(1)} \otimes I_{M_{l}} & \hat{D}_{R-i-2}^{(1)} \otimes I_{M_{l}} \\
\vdots & \vdots & \vdots & \ddots & \vdots & \vdots \\
O & O & O & \cdots & D_{0} \oplus S^{(l)} & \hat{D}_{1}^{(1)} \otimes I_{M_{l}} \\
O & O & O & \cdots & O & D(1) \oplus S^{(l)}
\end{array}\right) ;
$$

- $\mathfrak{f}_{N}^{(l)}$ is the square matrix of dimension $(R-N+1) \bar{W} M_{l}, \quad l=1,2$, having the same structure, as the matrix (10), but the matrix $D_{0}$ on diagonal blocks is replaced with $D_{0}+\hat{D}_{1}^{(2)}$.

Corollary 1. The macro-vectors $\tilde{\mathbf{p}}(0), \mathbf{p}(i, r), r=1,2$, satisfy the following system of equations:

$$
\begin{gathered}
\mathbf{0}=\tilde{\mathbf{p}}(0) D_{0}+\mathbf{p}(0,1) \boldsymbol{T}^{(1)}+\mathbf{p}(0,2) \boldsymbol{T}^{(2)}, \\
\mathbf{0}=\tilde{\mathbf{p}}(0) Z^{(1)}+\mathbf{p}(0,1)\left(\mathfrak{A}_{0}^{(1)}+\mathcal{B}_{0}^{(1,1)}\right)+\mathbf{p}(0,2) \mathcal{B}_{0}^{(2,1)}, \\
\mathbf{0}=\mathbf{p}(i, 1) \boldsymbol{A}_{i}^{(1)}+\sum_{l=0}^{i-1} \mathbf{p}(l, 1) \mathcal{D}_{l, i-l}^{(1)}, i=\overline{1, N}, \\
\quad \mathbf{0}=\mathbf{p}(i, 2) \mathfrak{A}_{i}^{(2)}+\tilde{\mathbf{p}}(0) \boldsymbol{Z}_{i+1}^{(2)}+\mathbf{p}(i+1,1) \mathcal{B}_{i+1}^{(1,2)}+ \\
\mathbf{p}(i+1,2) \mathcal{B}_{i+1}^{(2,2)}+\sum_{m=0}^{i-1} \mathbf{p}(m, 2) \mathcal{D}_{m, i-m}^{(2)}, i=\overline{1, N-1}, \\
\mathbf{0}=\mathbf{p}(N, 2) \mathfrak{A}_{N}^{(2)}+\tilde{\mathbf{p}}(0) \boldsymbol{Z}_{N+1}^{(2)}+\sum_{m=0}^{N-1} \mathbf{p}(m, 2) \mathcal{D}_{m, N-m}^{(2)} .
\end{gathered}
$$

Denote

$$
\begin{gathered}
\mathbf{p}(i)=(\mathbf{p}(i, 1), \mathbf{p}(i, 2)), i=\overline{0, N}, \\
\mathbf{p}=(\tilde{\mathbf{p}}(0), \mathbf{p}(0), \mathbf{p}(1), \ldots, \mathbf{p}(N)) .
\end{gathered}
$$

Corollary $\mathbf{2}$. The macro-vector $\mathbf{p}$ satisfies equation:

$$
\mathbf{p} Q=\mathbf{0},
$$

where $Q$ is generator of the Markov chain $\zeta_{t}, t \geq 0$, having the following structure:

$$
Q=\left(\begin{array}{ccccccc}
D_{0} & \mathcal{Z}_{0} & \mathcal{Z}_{1} & \mathcal{Z}_{2} & \mathcal{Z}_{3} & \cdots & \mathcal{Z}_{N} \\
\mathfrak{T} & \mathfrak{A}_{0} & \mathcal{D}_{0,1} & \mathfrak{D}_{0,2} & \mathcal{D}_{0,3} & \cdots & \mathfrak{D}_{0, N} \\
O & \mathfrak{B}_{1} & \mathfrak{A}_{1} & \mathfrak{D}_{1,1} & \mathfrak{D}_{1,2} & \cdots & \mathfrak{D}_{1, N-1} \\
O & O & \mathcal{B}_{2} & \mathfrak{f}_{2} & \mathfrak{D}_{2,1} & \cdots & \mathfrak{D}_{2, N-2} \\
\vdots & \vdots & \vdots & \vdots & \vdots & \ddots & \vdots \\
O & O & O & O & O & \cdots & \mathfrak{A}_{N}
\end{array}\right),
$$

where

$$
\begin{gathered}
\mathcal{T}=\left(\begin{array}{c}
\boldsymbol{T}^{(1)} \\
\boldsymbol{T}^{(2)}
\end{array}\right), \\
\mathfrak{f l}_{0}=\left(\begin{array}{cc}
\mathfrak{A}_{0}^{(1)}+\boldsymbol{B}_{0}^{(1,1)} & O \\
\mathbf{B}_{0}^{(2,1)} & \mathfrak{A}_{0}^{(2)}
\end{array}\right),
\end{gathered}
$$

$$
\begin{gathered}
\mathfrak{A}_{i}=\operatorname{diag}\left\{\boldsymbol{A}_{i}^{(1)}, \mathfrak{A}_{i}^{(2)}\right\}, i=\overline{1, N}, \\
\boldsymbol{B}_{i}=\left(\begin{array}{ll}
O & \boldsymbol{B}_{i}^{(1,2)} \\
O & \mathcal{B}_{i}^{(2,2)}
\end{array}\right), i=\overline{1, N},
\end{gathered}
$$

$$
\begin{gathered}
\mathcal{D}_{i, j}=\operatorname{diag}\left\{\mathfrak{D}_{i, j}^{(1)}, \mathfrak{D}_{i, j}^{(2)}\right\}, j=\overline{1, N-i}, i=\overline{0, N-1}, \\
\boldsymbol{Z}_{0}=\left(\begin{array}{ll}
\boldsymbol{Z}^{(1)} & \boldsymbol{Z}_{1}^{(2)}
\end{array}\right), \\
\boldsymbol{Z}_{i}=\left(\begin{array}{ll}
O & \boldsymbol{Z}_{i+1}^{(2)}
\end{array}\right), i=\overline{1, N},
\end{gathered}
$$

$\operatorname{diag}\left\{C_{1}, \ldots, C_{N}\right\}$ denotes the block diagonal matrix with the diagonal entries $\left\{C_{1}, \ldots, C_{N}\right\}$.

Direct solution of the system (16) is possible as it is the finite system of linear algebraic equations with normalization condition

$$
\tilde{\mathbf{p}}(0) \mathbf{e}+\sum_{i=0}^{N} \mathbf{p}(i) \mathbf{e}=1
$$

However, the blocking matrix (17) (generator of the process $\zeta_{t}, t \geq 0$ ) has only non-zero blocks below the sub-diagonal. So, the effective and stable algorithm for solving system (16), (18), which is presented in [11], [12], can be applied for solving this system.

Having the stationary distribution been computed we can easy calculate, e.g., the mean queue lengths and their variations.

Corollary 3. Average number $L_{1}^{(l)}$ of type-l customers in the buffer is calculated by

$$
L_{1}^{(l)}=\sum_{r=1}^{2} \sum_{i_{2}=0}^{N} \sum_{i_{1}=0}^{R-i_{2}} i_{l} \mathbf{p}\left(i_{2}, i_{1}, r\right), l=1,2 .
$$

Variation $V^{(l)}$ of the number of type- $l$ customers in the buffer is calculated by

$$
V^{(l)}=\sum_{r=1}^{2} \sum_{i_{2}=0}^{N} \sum_{i_{1}=0}^{R-i_{2}} i_{l}^{2} \mathbf{p}\left(i_{2}, i_{1}, r\right)-\left(L_{1}^{(l)}\right)^{2}, l=1,2 .
$$

\section{LOSS PROBABILITY}

We will assume below that customers of the same priority class are served according to FIFO (first in - first out) discipline and the customers in an arriving batch of size $k$ are 
numbered in such a way as an arbitrary customer gets the number $m, m=\overline{1, k}, k \geq 1$, with equal probability $\frac{1}{k}$.

Having the stationary distribution of the Markov chain $\zeta_{t}, t \geq 0$, been computed, different performance measures of the system can be calculated and optimization problems can be solved. In particular, probabilities $P_{\text {loss }}^{(l)}$ that the arbitrary type$l$ customer, $l=1,2$, is lost are calculated as given in the following statement.

Theorem 2. The loss probabilities $P_{\text {loss }}^{(l)}, \quad l=1,2$, are calculated by

$$
\begin{gathered}
P_{l o s s}^{(1)}=\lambda_{1}^{-1}\left(\mathbf{p}(0,0,0) \sum_{k=R+2}^{\infty}(k-R-1) D_{k}^{(1)} \mathbf{e}+\right. \\
\left.\sum_{l=1}^{2} \sum_{i_{2}=0}^{N} \sum_{i_{1}=0}^{R-i_{2}} \mathbf{p}\left(i_{2}, i_{1}, l\right) \sum_{k=R-i_{2}-i_{1}+1}^{\infty}\left(k-R+i_{2}+i_{1}\right) D_{k}^{(1)} \otimes I_{M_{l}} \mathbf{e}\right), \\
P_{\text {loss }}^{(2)}=\lambda_{2}^{-1}\left(\mathbf{p}(0,0,0) \sum_{k=N+2}^{\infty}(k-N-1) D_{k}^{(2)} \mathbf{e}+\right. \\
\sum_{l=1}^{2} \sum_{i_{2}=0}^{N} \sum_{i_{1}=0}^{R-i_{2}} \mathbf{p}\left(i_{2}, i_{1}, l\right) \sum_{k=\min \left\{N, R-i_{1}\right\}-i_{2}+1}^{\infty}(k- \\
\left.\left.\min \left\{N, R-i_{1}\right\}-i_{2}\right) D_{k}^{(2)} \otimes I_{M_{l}} \mathbf{e}\right) .
\end{gathered}
$$

Proof. The statement of the theorem is proved using the formula of total probability and the following evident assertions.

- Probability that arbitrary type- $l$ customer arrives during small time interval $(u, u+\Delta t)$ in a batch consisting of $k$ customers and a state of the directing process $v_{t}, t \geq 0$, of the BMMAP changes to the value $v^{\prime}$, conditional it had a value $v$ at epoch $u$, is the $\left(v, v^{\prime}\right)$ th entry of the matrix

$$
\lambda_{l}^{-1} k D_{k}^{(l)} \Delta t, k \geq 1, l=1,2
$$

- Due to assumption that an arbitrary customer arriving in a batch of size $k$ gets the number $m, m=\overline{1, k}$, with equal probability $\frac{1}{k}$, it is clear that: $(i)$ arbitrary type- 2 customer arriving in a batch consisting of $k$ customers is lost with probability $\frac{k-(N+1)}{k}, k>N+1$, if the batch meets the empty system and is lost with probability $\frac{k-\min \left\{N, R-i_{1}\right\}-i_{2}}{k}$ if the batch meets the system in the state $\left(i_{2}, i_{1}, l\right) ;(i i)$ arbitrary type-1 customer arriving in a batch consisting of $k$ customers is lost with probability $\frac{k-(R+1)}{k}, k>R+1$, if the batch meets the empty system and is lost with probability $\frac{k-\left(R-i_{2}-i_{1}\right)}{k}$ if the batch meets the system in the state $\left(i_{2}, i_{1}, l\right)$.

\section{WAITING TIME DisTRIBUTION}

Denote $W_{2}(x)$ waiting time distribution function for type-2 (real-time) flow. Let $\omega_{2}(v)$ be its Laplace-Stieltjes transform $\omega_{2}(v)=\int_{0}^{\infty} e^{-v x} d W_{2}(x), \operatorname{Re} v>0, W_{1}^{(2)}$ be the mean waiting time, $W_{2}^{(2)}$ be the second initial moment of distribution function and $J_{2}$ be a jitter for type-2 customers.

Theorem 3. Laplace-Stieltjes transform $\omega_{2}(v)$ of waiting time distribution function for type-2 customers is calculated as follows:

$$
\begin{gathered}
\omega_{2}(v)=\lambda_{2}^{-1}\left(P_{l o s s}^{(2)}+\mathbf{p}(0,0,0) \sum_{k=1}^{\infty} D_{k}^{(2)} \mathbf{e} \times\right. \\
\times \sum_{i=0}^{\min \{k-1, N\}}\left(\boldsymbol{\beta}_{2}\left(v I-S^{(2)}\right)^{-1} S_{0}^{(2)}\right)^{i}+ \\
\sum_{l=1}^{2} \sum_{i_{2}=0}^{N-1} \sum_{i_{1}=0}^{R-i_{2}-1} \mathbf{p}\left(i_{2}, i_{1}, l\right) \sum_{k=1}^{\infty} D_{k}^{(2)} \mathbf{e} \otimes \boldsymbol{Y}^{(l)}(v) \times \\
\times \sum_{m=0}^{\min \left\{k, \min \left\{N, R-i_{1}\right\}-i_{2}\right\}-1}\left(\boldsymbol{\beta}_{2} \times\right. \\
\left.\left.\times\left(v I-S^{(2)}\right)^{-1} S_{0}^{(2)}\right)^{i_{2}+m}\right),
\end{gathered}
$$

where

$$
\boldsymbol{\gamma}^{(l)}(v)=\left(v I-S^{(l)}\right)^{-1} S_{0}^{(l)}, l=1,2 .
$$

Proof. To prove the theorem, we use the method of collective marks (method of catastrophes). We temporarily assume that the parameter $v$ is real and interpret it as an intensity of some imaginary stationary Poisson flow of catastrophes. Thus, $\omega_{2}(v)$ has sense of probability that no one catastrophe arrives during the waiting time of an arbitrary type- 2 customer. Then, we use formula of total probability. We take into account formula (21) and information that the entries of the vector $\boldsymbol{V}^{(l)}(v)$ define probability of no catastrophe arrival during the residual service of a type- $l$ customer being in service at an arbitrary customer arrival epoch and the function $\boldsymbol{\beta}_{2}\left(v I-S^{(2)}\right)^{-1} S_{0}^{(2)}$ define probability of no catastrophe arrival during the total service time of type-2 customer. Sure, no one catastrophe arrives during the waiting time of an arbitrary type- 2 customer if it is lost. As the result, we have formula (22) been proven.

Corollary 4. The mean waiting time $W_{1}^{(2)}$, the second initial moment $W_{2}^{(2)}$ of distribution function and a jitter $J_{2}$ for type- 2 customers are calculated by 


$$
\begin{aligned}
W_{1}^{(2)}= & -\omega_{2}^{\prime}(0)=\lambda_{2}^{-1}\left(\mathbf{p}(0,0,0) \sum_{k=1}^{\infty} D_{k}^{(2)} \mathbf{e} \boldsymbol{\beta}_{1}^{(2)} \times\right. \\
& \times \frac{\min \{k, N+1\} \min \{k-1, N\}}{2}+
\end{aligned}
$$$$
\sum_{l=1}^{2} \sum_{i_{2}=0}^{N-1} \sum_{i_{1}=0}^{R-i_{2}-1} \mathbf{p}\left(i_{2}, i_{1}, l\right) \sum_{k=1}^{\infty} D_{k}^{(2)} \otimes I_{M_{l}} \times
$$$$
\times\left(\mathbf{e} \otimes \overline{\boldsymbol{V}}^{(l)}+\mathbf{e} \boldsymbol{\beta}_{1}^{(2)} \min \left\{k, \min \left\{N, R-i_{1}\right\}-i_{2}\right\} \times\right.
$$$$
\left.\times\left[i_{2}+\frac{1}{2} \min \left\{k, \min \left\{N, R-i_{1}\right\}-i_{2}\right\}-1\right]\right) \text {, }
$$$$
W_{2}^{(2)}=-\omega_{2}^{\prime \prime}(0)=\lambda_{2}^{-1}\left(\mathbf{p}(0,0,0) \sum_{k=1}^{\infty} D_{k}^{(2)} \mathbf{e} \times\right.
$$$$
\times \sum_{i=0}^{\min \{k-1, N\}}\left(i \boldsymbol{\beta}_{2}^{(2)}+\frac{i(i-1)}{2}\left(\boldsymbol{\beta}_{1}^{(2)}\right)^{2}\right)+
$$$$
\sum_{l=1}^{2} \sum_{i_{2}=0}^{N-1} \sum_{i_{1}=0}^{R-i_{2}-1} \mathbf{p}\left(i_{2}, i_{1}, l\right) \sum_{k=1}^{\infty} D_{k}^{(2)} \otimes I_{M_{l}}\left(\mathbf{e} \otimes \overline{\overline{\boldsymbol{Y}}}^{(l)}+\right.
$$$$
\sum_{m=i_{2}}^{\min \left\{k+i_{2}, \min \left\{N, R-i_{1}\right\}\right\}-1}\left(2 \mathbf{e} \otimes \overline{\boldsymbol{Y}}^{(l)} \boldsymbol{\beta}_{1}^{(2)} m+m \boldsymbol{\beta}_{2}^{(2)} \mathbf{e}+\right.
$$$$
\left.\left.\left.m(m-1)\left(\boldsymbol{\beta}_{1}^{(2)}\right)^{2} \mathbf{e}\right)\right)\right)
$$$$
J_{2}=W_{2}^{(2)}-\left(W_{1}^{(2)}\right)^{2}
$$

where

$$
\begin{gathered}
\overline{\boldsymbol{V}}^{(l)}=\left(-S^{(l)}\right)^{-1} \mathbf{e}, \overline{\overline{\boldsymbol{V}}}^{(l)}=2\left(-S^{(l)}\right)^{-2} \mathbf{e}, \\
\boldsymbol{\beta}_{1}^{(l)}=\boldsymbol{\beta}^{(l)}\left(-S^{(l)}\right)^{-1} \mathbf{e}, \boldsymbol{\beta}_{2}^{(l)}=2 \boldsymbol{\beta}^{(l)}\left(-S^{(l)}\right)^{-2} \mathbf{e} .
\end{gathered}
$$

Waiting time distribution $W_{2}(x)$ defines probability that waiting time of an arbitrary type-2 customer is less or equal to $x$. Denote by $\tilde{W}_{2}(x)$ the waiting time distribution for an arbitrary type-2 customer which was not lost due to the buffer overfull. It is easy to see that the distribution functions $W_{2}(x)$ and $\tilde{W}_{2}(x)$ are related as follows:

$$
\widetilde{W}_{2}(x)=\frac{W_{2}(x)-P_{\text {loss }}^{(2)}}{1-P_{\text {loss }}^{(2)}} .
$$

So, the following corollary is obvious.

Corollary 5. The mean waiting time $\widetilde{W}_{1}^{(2)}$, the second initial moment $\widetilde{W}_{2}^{(2)}$ of distribution function and a jitter $\widetilde{J}_{2}$ for type-2 customers are calculated by

$$
\begin{gathered}
\tilde{W}_{1}^{(2)}=\frac{W_{1}^{(2)}}{1-P_{\text {loss }}^{(2)},} \\
\tilde{W}_{2}^{(2)}=\frac{W_{2}^{(2)}}{1-P_{\text {loss }}^{(2)}}, \\
\widetilde{J}_{2}=\widetilde{W}_{2}^{(2)}-\left(W_{1}^{(2)}\right)^{2} .
\end{gathered}
$$

\section{NUMERICAL EXAMPLES}

Analytical results, which are presented above, have transparent algorithmic form. So, their realization on computer does not meet essential problems. Realization was developed in frameworks of software "SIRIUS++", see, e.g. [8].

Basic parameters of the buffer, flow and service processes are the following.

We assume that capacity $R$ of a buffer is equal to 32 and the number $N$ of places available in a buffer for priority customers is equal to 6 .

Type-1 packets can arrive in batches consisting of $1,2,3$ or 4 packets and type-2 customers can arrive in batches consisting of 1 or 2 packets. The matrices $D_{0}, D_{k}^{(l)}$, which define the $B M M A P$, are given by

$$
\begin{gathered}
D_{0}=\left(\begin{array}{cc}
-0.1646 & 0 \\
0 & -0.1651
\end{array}\right), \\
D_{1}^{(1)}=\left(\begin{array}{cc}
0.002 & 0.0022 \\
0.0019 & 0.002
\end{array}\right), D_{2}^{(1)}=\left(\begin{array}{cc}
0.004 & 0.0031 \\
0.0038 & 0.0042
\end{array}\right), \\
D_{3}^{(1)}=\left(\begin{array}{ll}
0.0055 & 0.0053 \\
0.0051 & 0.0059
\end{array}\right), D_{4}^{(1)}=\left(\begin{array}{cc}
0.006 & 0.0061 \\
0.0062 & 0.0059
\end{array}\right), \\
D_{1}^{(2)}=\left(\begin{array}{ll}
0.0301 & 0.0301 \\
0.0302 & 0.0299
\end{array}\right), D_{2}^{(2)}=\left(\begin{array}{cc}
0.035 & 0.0352 \\
0.0351 & 0.0349
\end{array}\right) .
\end{gathered}
$$

These matrices provide the values of intensity $\lambda_{l}$ of type- $l$ customers arrival as

$$
\lambda_{1}=0.1, \lambda_{2}=0.2
$$

Service time distribution of type-1 packets is characterized by irreducible representation $\left(\boldsymbol{\beta}_{1}, S^{(1)}\right)$, where

$$
\boldsymbol{\beta}_{1}=\left(\begin{array}{lll}
0.3 & 0.2 & 0.5
\end{array}\right), S^{(1)}=\left(\begin{array}{ccc}
-1.7 & 1.4 & 0.1 \\
1.3 & -2.6 & 1.2 \\
1.7 & 1.5 & -3.4
\end{array}\right) \text {. }
$$

Mean service time $\boldsymbol{\beta}_{1}^{(1)}$ is equal to 6 .

Service time distribution of type-2 packets is characterized by irreducible representation $\left(\boldsymbol{\beta}_{2}, S^{(2)}\right)$, where

$$
\boldsymbol{\beta}_{2}=\left(\begin{array}{ll}
0.4 & 0.6
\end{array}\right), S^{(2)}=\left(\begin{array}{cc}
-1.4 & 1.1 \\
1.2 & -1.9
\end{array}\right) \text {. }
$$

Mean service time $\boldsymbol{\beta}_{1}^{(2)}$ is equal to 2 .

Five experiments were made to give insight into dependence of the main performance measures of the system on its parameters.

Experiment 1. The aim of this experiment is to analyze dependence of the main performance measures of the system on the average intensity $\lambda_{1}$ of type-1 packets arrival.

In the Table 1 , values of loss probabilities $P_{\text {loss }}^{(1)}, P_{\text {loss }}^{(2)}$, mean waiting time of type-2 packets $W_{1}^{(2)}$, second moment $W_{2}^{(2)}$ of type-2 packets waiting time distribution and jitter $J_{2}$ as well as the analogous values $\tilde{W}_{1}^{(2)}, \widetilde{W}_{2}^{(2)}, \widetilde{J}_{2}$ for waiting 
TABLE I

Dependence of the Main Performance Measures on the Average Intensity $\lambda_{1}$ of Non-Priority Packets Arrival

\begin{tabular}{|c|c|c|c|c|c|c|c|c|c|c|}
\hline$\lambda_{1}$ & $P_{\text {loss }}^{(1)}$ & $P_{\text {loss }}^{(2)}$ & $W_{1}^{(2)}$ & $W_{2}^{(2)}$ & $J_{2}$ & $\widetilde{W}_{1}^{(2)}$ & $\widetilde{W}_{2}^{(2)}$ & $\widetilde{J}_{2}$ & $L_{1}^{(1)}$ & $L_{1}^{(2)}$ \\
\hline 0.020 & $3.1 \mathrm{e}-08$ & 0.0176 & 2.9458 & 31.1340 & 22.4562 & 2.9985 & 31.6910 & 22.6999 & 0.4381 & 0.6965 \\
\hline 0.069 & 0.0031 & 0.0401 & 4.7717 & 61.8983 & 39.1296 & 4.9708 & 64.4822 & 39.7729 & 4.6222 & 1.1780 \\
\hline 0.118 & 0.1379 & 0.1167 & 5.2634 & 70.9553 & 43.2517 & 5.9589 & 80.3308 & 44.8224 & 19.7817 & 1.3237 \\
\hline 0.167 & 0.3307 & 0.2335 & 4.3733 & 57.6298 & 38.5040 & 5.7055 & 75.1853 & 42.6323 & 26.2667 & 1.1101 \\
\hline 0.216 & 0.4563 & 0.3169 & 3.7511 & 48.5265 & 34.4556 & 5.4914 & 71.0392 & 40.8841 & 28.0097 & 0.9555 \\
\hline 0.265 & 0.5386 & 0.3863 & 3.2731 & 41.7131 & 31.0001 & 5.3334 & 67.9710 & 39.5254 & 28.9130 & 0.8343 \\
\hline 0.314 & 0.5994 & 0.4384 & 2.9395 & 37.0724 & 28.4319 & 5.2338 & 66.0083 & 38.6155 & 29.4175 & 0.7483 \\
\hline 0.363 & 0.6437 & 0.4912 & 2.6198 & 32.7167 & 25.8532 & 5.1487 & 64.2975 & 37.7883 & 29.8407 & 0.6647 \\
\hline 0.412 & 0.6807 & 0.5242 & 2.4292 & 30.1673 & 24.2660 & 5.1061 & 63.4098 & 37.3372 & 30.0684 & 0.6143 \\
\hline 0.461 & 0.7098 & 0.5553 & 2.2575 & 27.9002 & 22.8037 & 5.0765 & 62.7391 & 36.9681 & 30.2669 & 0.5655 \\
\hline 0.510 & 0.7345 & 0.5820 & 2.1136 & 26.0223 & 21.5551 & 5.0562 & 62.2523 & 36.6871 & 30.4213 & 0.5271 \\
\hline 0.559 & 0.7547 & 0.6042 & 1.9966 & 24.5114 & 20.5250 & 5.0441 & 61.9241 & 36.4815 & 30.5419 & 0.4958 \\
\hline 0.608 & 0.7722 & 0.6245 & 1.8913 & 23.1630 & 19.5858 & 5.0371 & 61.6881 & 36.3161 & 30.6469 & 0.4675 \\
\hline 0.657 & 0.7875 & 0.6410 & 1.8074 & 22.0952 & 18.8284 & 5.0344 & 61.5440 & 36.1988 & 30.7284 & 0.4450 \\
\hline 0.706 & 0.8005 & 0.6593 & 1.7156 & 20.9353 & 17.9918 & 5.0353 & 61.4430 & 36.0892 & 30.8158 & 0.4203 \\
\hline 0.755 & 0.8122 & 0.6735 & 1.6449 & 20.0464 & 17.3406 & 5.0381 & 61.3973 & 36.0152 & 30.8810 & 0.4013 \\
\hline 0.804 & 0.8224 & 0.6884 & 1.5718 & 19.1314 & 16.6610 & 5.0443 & 61.3993 & 35.9540 & 30.9475 & 0.3816 \\
\hline 0.853 & 0.8315 & 0.7021 & 1.5052 & 18.3036 & 16.0378 & 5.0525 & 61.4381 & 35.9100 & 31.0066 & 0.3637 \\
\hline 0.902 & 0.8398 & 0.7142 & 1.4465 & 17.5754 & 15.4831 & 5.0618 & 61.5033 & 35.8810 & 31.0577 & 0.3480 \\
\hline 0.951 & 0.8472 & 0.7252 & 1.3936 & 16.9214 & 14.9794 & 5.0716 & 61.5826 & 35.8612 & 31.1027 & 0.3339 \\
\hline 1.000 & 0.8526 & 0.7635 & 1.2115 & 14.6866 & 13.2190 & 5.1217 & 62.0908 & 35.8592 & 31.2513 & 0.2868 \\
\hline
\end{tabular}

time distribution and jitter of type-2 packets, which are not lost, the average number $L_{1}^{(1)}$ of type-1 customers in the buffer, the average number $L_{1}^{(2)}$ of type-2 customers in the buffer, are presented along with the corresponding value of the average intensity $\lambda_{1}$. Intensity $\lambda_{2}$ is equal to 0.2 .

Figures 2 and 3 present the dependence of the characteristics $P_{\text {loss }}^{(1)}, P_{\text {loss }}^{(2)}, \widetilde{J}_{2}$ on the average intensity $\lambda_{1}$ in the graphical form.

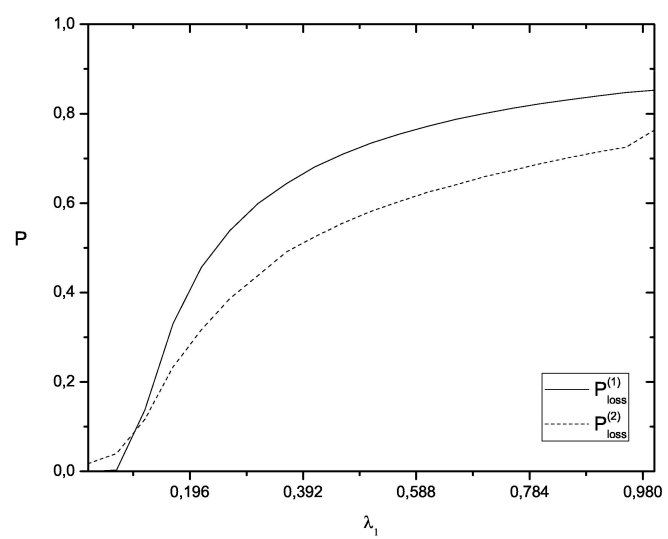

Fig. 2. Dependence of the loss probabilities $P_{\text {loss }}^{(1)}, P_{\text {loss }}^{(2)}$ on the average intensity $\lambda_{1}$ of non-priority packets

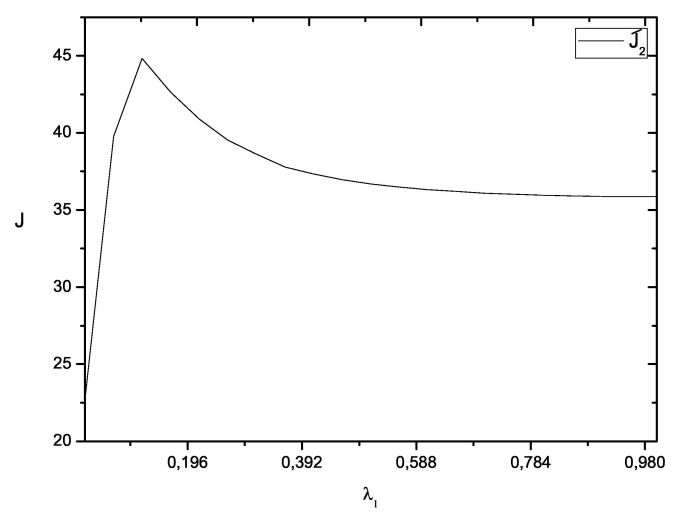

Fig. 3. Dependence of the jitter $\widetilde{J}_{2}$ of priority packets on the average intensity $\lambda_{1}$ of non-priority packets

Experiment 2. The aim of this experiment is to analyze dependence of the main performance measures of the system on the average intensity $\lambda_{2}$ of type- 2 packets arrival.

In the Table 2, values of loss probabilities $P_{\text {loss }}^{(1)}, P_{\text {loss }}^{(2)}$, mean waiting time of type-2 packets $W_{1}^{(2)}$, second moment $W_{2}^{(2)}$ of type-2 packets waiting time distribution and jitter $J_{2}$ as well as the analogous values $\widetilde{W}_{1}^{(2)}, \widetilde{W}_{2}^{(2)}, \widetilde{J}_{2}$ for waiting 
TABLE II

Dependence of the Main Performance Measures on the Average Intensity $\lambda_{2}$ of Priority Packets Arrival

\begin{tabular}{|c|c|c|c|c|c|c|c|c|c|c|}
\hline$\lambda_{2}$ & $P_{\text {loss }}^{(1)}$ & $P_{\text {loss }}^{(2)}$ & $W_{1}^{(2)}$ & $W_{2}^{(2)}$ & $J_{2}$ & $\widetilde{W}_{1}^{(2)}$ & $\widetilde{W}_{2}^{(2)}$ & $\widetilde{J}_{2}$ & $L_{1}^{(1)}$ & $L_{1}^{(2)}$ \\
\hline 0.020 & 0.0003 & 0.0002 & 3.1825 & 38.6870 & 28.5589 & 3.1831 & 38.6941 & 28.5623 & 2.7645 & 0.0090 \\
\hline 0.069 & 0.0023 & 0.0039 & 4.1793 & 53.3927 & 35.9261 & 4.1959 & 53.6042 & 35.9990 & 4.5414 & 0.3688 \\
\hline 0.118 & 0.0092 & 0.0207 & 4.6195 & 61.8992 & 40.5594 & 4.7173 & 63.2096 & 40.9567 & 7.0767 & 0.7413 \\
\hline 0.167 & 0.0284 & 0.0533 & 4.9933 & 68.7129 & 43.7795 & 5.2743 & 72.5799 & 44.7612 & 10.8407 & 1.1355 \\
\hline 0.216 & 0.0690 & 0.1030 & 5.1339 & 71.5784 & 45.2218 & 5.7233 & 79.7959 & 47.0402 & 15.4958 & 1.4979 \\
\hline 0.265 & 0.1300 & 0.1593 & 5.0461 & 70.2211 & 44.7581 & 6.0020 & 83.5231 & 47.4995 & 19.7999 & 1.7780 \\
\hline 0.314 & 0.1983 & 0.2151 & 4.8297 & 66.6055 & 43.2799 & 6.1534 & 84.8617 & 46.9969 & 22.7659 & 1.9857 \\
\hline 0.363 & 0.2635 & 0.2658 & 4.5820 & 62.4736 & 41.4787 & 6.2409 & 85.0918 & 46.1427 & 24.5378 & 2.1473 \\
\hline 0.412 & 0.3222 & 0.3107 & 4.3473 & 58.5576 & 39.6583 & 6.3070 & 84.9533 & 45.1756 & 25.5819 & 2.2812 \\
\hline 0.461 & 0.3740 & 0.3503 & 4.1323 & 55.0180 & 37.9420 & 6.3602 & 84.6806 & 44.2283 & 26.2238 & 2.3973 \\
\hline 0.510 & 0.4205 & 0.3858 & 3.9361 & 51.7987 & 36.3059 & 6.4081 & 84.3306 & 43.2664 & 26.6549 & 2.4966 \\
\hline 0.559 & 0.4619 & 0.4176 & 3.7566 & 48.8866 & 34.7744 & 6.4499 & 83.9362 & 42.3344 & 26.9558 & 2.5829 \\
\hline 0.608 & 0.4994 & 0.4462 & 3.5934 & 46.2341 & 33.3214 & 6.4885 & 83.4835 & 41.3823 & 27.1808 & 2.6578 \\
\hline 0.657 & 0.5321 & 0.4727 & 3.4342 & 43.7702 & 31.9765 & 6.5129 & 83.0091 & 40.5916 & 27.3454 & 2.7236 \\
\hline 0.706 & 0.5625 & 0.4965 & 3.2925 & 41.5198 & 30.6792 & 6.5396 & 82.4673 & 39.7004 & 27.4825 & 2.7797 \\
\hline 0.755 & 0.5900 & 0.5183 & 3.1608 & 39.4412 & 29.4507 & 6.5622 & 81.8851 & 38.8228 & 27.5945 & 2.8280 \\
\hline 0.804 & 0.6148 & 0.5386 & 3.0352 & 37.4922 & 28.2799 & 6.5781 & 81.2563 & 37.9851 & 27.6883 & 2.8689 \\
\hline 0.853 & 0.6371 & 0.5572 & 2.9049 & 35.6996 & 27.1864 & 6.5899 & 80.6298 & 37.2031 & 27.7653 & 2.9177 \\
\hline 0.902 & 0.6573 & 0.5746 & 2.8053 & 34.0057 & 26.1362 & 6.5951 & 79.9462 & 36.4510 & 27.8336 & 2.9347 \\
\hline 0.951 & 0.6756 & 0.5909 & 2.6982 & 32.4139 & 25.1339 & 6.5951 & 79.2290 & 35.7342 & 27.8941 & 2.9592 \\
\hline 1.000 & 0.6913 & 0.6064 & 2.5922 & 30.9211 & 24.2015 & 6.5858 & 78.5578 & 35.1852 & 27.9423 & 2.9812 \\
\hline
\end{tabular}

time distribution and jitter of type- 2 packets, which are not lost, the average number $L_{1}^{(1)}$ of type-1 customers in the buffer, the average number $L_{1}^{(2)}$ of type-2 customers in the buffer, are presented along with the corresponding value of the average intensity $\lambda_{2}$. Intensity $\lambda_{1}$ is equal to 0.1 .

Figures 4 and 5 present the dependence of the characteristics $P_{\text {loss }}^{(1)}, P_{\text {loss }}^{(2)}, \widetilde{J}_{2}$ on the average intensity $\lambda_{2}$ in the graphical form.

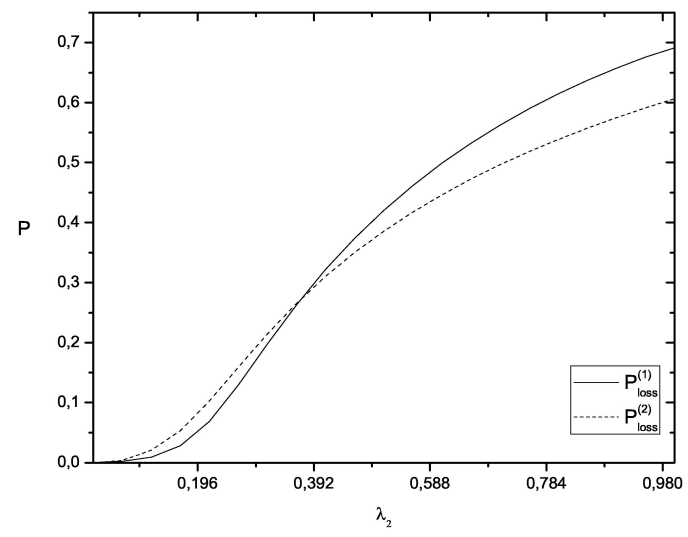

Fig. 4. Dependence of the loss probabilities $P_{\text {loss }}^{(1)}, P_{\text {loss }}^{(2)}$ on the intensity $\lambda_{2}$ of priority packets

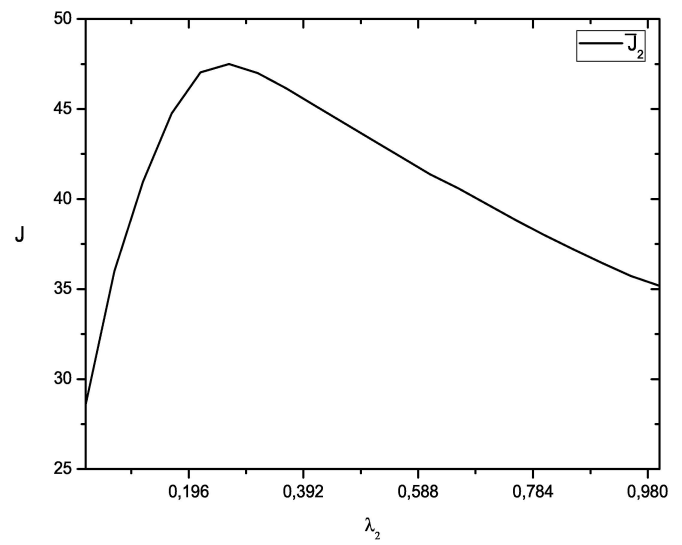

Fig. 5. Dependence of the jitter $\widetilde{J}_{2}$ of priority packets on the intensity $\lambda_{2}$ of priority packets

Experiment 3. The aim of this experiment is to analyze dependence of the main performance measures of the system on the capacity $R$ of the buffer. Starting from the value $R=6$, we increase the buffer capacity one-by-one.In the Table 3, values of loss probabilities $P_{\text {loss }}^{(1)}, P_{\text {loss }}^{(2)}$, mean waiting time of type-2 packets $W_{1}^{(2)}$, second moment $W_{2}^{(2)}$ of type-2 packets waiting time distribution and jitter $J_{2}$ 
TABLE III

Dependence of the Main Performance Measures on the Total Capacity $R$ of the Buffer

\begin{tabular}{|c|c|c|c|c|c|c|c|c|c|c|}
\hline$R$ & $P_{\text {loss }}^{(1)}$ & $P_{\text {loss }}^{(2)}$ & $W_{1}^{(2)}$ & $W_{2}^{(2)}$ & $J_{2}$ & $\widetilde{W}_{1}^{(2)}$ & $\widetilde{W}_{2}^{(2)}$ & $\widetilde{J}_{2}$ & $L_{1}^{(1)}$ & $L_{1}^{(2)}$ \\
\hline 6 & 0.2556 & 0.1701 & 3.4224 & 40.7038 & 28.9910 & 4.1241 & 49.0493 & 32.0416 & 1.6252 & 0.8553 \\
\hline 7 & 0.2273 & 0.1539 & 3.7281 & 45.6005 & 31.7017 & 4.4065 & 53.8980 & 34.4810 & 2.0319 & 0.9329 \\
\hline 8 & 0.2040 & 0.1413 & 3.9792 & 49.6531 & 33.8193 & 4.6338 & 57.8223 & 36.3498 & 2.4614 & 0.9961 \\
\hline 9 & 0.1845 & 0.1314 & 4.1836 & 52.9718 & 35.4689 & 4.8167 & 60.9877 & 37.7868 & 2.9090 & 1.0473 \\
\hline 10 & 0.1682 & 0.1237 & 4.3510 & 55.6921 & 36.7612 & 4.9649 & 63.5508 & 38.9002 & 3.3700 & 1.0892 \\
\hline 11 & 0.1543 & 0.1174 & 4.4899 & 57.9563 & 37.7972 & 5.0869 & 65.6622 & 39.7861 & 3.8404 & 1.1239 \\
\hline 12 & 0.1424 & 0.1121 & 4.6072 & 59.8689 & 38.6430 & 5.1890 & 67.4292 & 40.5039 & 4.3180 & 1.1532 \\
\hline 13 & 0.1322 & 0.1077 & 4.7069 & 61.4955 & 39.3403 & 5.2752 & 68.9197 & 41.0921 & 4.8010 & 1.1782 \\
\hline 14 & 0.1233 & 0.1040 & 4.7928 & 62.8958 & 39.9244 & 5.3489 & 70.1934 & 41.5822 & 5.2880 & 1.1996 \\
\hline 15 & 0.1156 & 0.1007 & 4.8677 & 64.1163 & 40.4216 & 5.4128 & 71.2961 & 41.9976 & 5.7782 & 1.2183 \\
\hline 16 & 0.1087 & 0.0978 & 4.9335 & 65.1884 & 40.8490 & 5.4686 & 72.2588 & 42.3533 & 6.2708 & 1.2347 \\
\hline 17 & 0.1026 & 0.0953 & 4.9917 & 66.1374 & 41.2200 & 5.5177 & 73.1061 & 42.6611 & 6.7654 & 1.2492 \\
\hline 18 & 0.0972 & 0.0931 & 5.0437 & 66.9837 & 41.5451 & 5.5613 & 73.8580 & 42.9301 & 7.2617 & 1.2622 \\
\hline 19 & 0.0924 & 0.0911 & 5.0903 & 67.7434 & 41.8322 & 5.6002 & 74.5298 & 43.1671 & 7.7595 & 1.2738 \\
\hline 20 & 0.0880 & 0.0892 & 5.1324 & 68.4290 & 42.0876 & 5.6353 & 75.1337 & 43.3776 & 8.2585 & 1.2844 \\
\hline 21 & 0.0840 & 0.0876 & 5.1706 & 69.0509 & 42.3163 & 5.6669 & 75.6793 & 43.5656 & 8.7587 & 1.2939 \\
\hline 22 & 0.0803 & 0.0861 & 5.2053 & 69.6177 & 42.5221 & 5.6956 & 76.1749 & 43.7347 & 9.2599 & 1.3026 \\
\hline 23 & 0.0770 & 0.0847 & 5.2372 & 70.1363 & 42.7082 & 5.7218 & 76.6269 & 43.8874 & 9.7620 & 1.3105 \\
\hline 24 & 0.0740 & 0.0834 & 5.2664 & 70.6127 & 42.8775 & 5.7459 & 77.0409 & 44.0261 & 10.2651 & 1.3178 \\
\hline 25 & 0.0711 & 0.0823 & 5.2934 & 71.0518 & 43.0320 & 5.7679 & 77.4215 & 44.1526 & 10.7689 & 1.3245 \\
\hline 26 & 0.0685 & 0.0812 & 5.3183 & 71.4578 & 43.1735 & 5.7883 & 77.7726 & 44.2684 & 11.2734 & 1.3308 \\
\hline 27 & 0.0661 & 0.0802 & 5.3414 & 71.8344 & 43.3037 & 5.8071 & 78.0975 & 44.3748 & 11.7787 & 1.3365 \\
\hline 28 & 0.0639 & 0.0793 & 5.3629 & 72.1846 & 43.4238 & 5.8246 & 78.3990 & 44.4729 & 12.2847 & 1.3419 \\
\hline 29 & 0.0618 & 0.0784 & 5.3830 & 72.5111 & 43.5349 & 5.8409 & 78.6795 & 44.5637 & 12.7913 & 1.3469 \\
\hline 30 & 0.0598 & 0.0776 & 5.4017 & 72.8162 & 43.6380 & 5.8560 & 78.9412 & 44.6479 & 13.2985 & 1.3516 \\
\hline 31 & 0.0580 & 0.0768 & 5.4192 & 73.1020 & 43.7340 & 5.8702 & 79.1859 & 44.7262 & 13.8063 & 1.3559 \\
\hline 32 & 0.0563 & 0.0761 & 5.4357 & 73.3702 & 43.8235 & 5.8835 & 79.4152 & 44.7992 & 14.3147 & 1.3601 \\
\hline
\end{tabular}

as well as the analogous values $\widetilde{W}_{1}^{(2)}, \widetilde{W}_{2}^{(2)}, \widetilde{J}_{2}$ for waiting time distribution and jitter of type-2 packets, which are not lost, the average number $L_{1}^{(1)}$ of type- 1 customers in the buffer, the average number $L_{1}^{(2)}$ of type-2 customers in the buffer, are presented along with the corresponding value of $R$.

Figures 6 and 7 present the dependence of the characteristics $P_{\text {loss }}^{(1)}, P_{\text {loss }}^{(2)}, \widetilde{J}_{2}$ on the buffer capacity $R$ in the graphical form.

Experiment 4. The aim of this experiment is to analyze dependence of the main performance measures of the system on the number $N$ of places in a buffer which are available for priority packets. The value of the total buffer capacity $R$ is assumed to be equal to 32 . Starting from the value $N=6$, we increase the buffer capacity available for the priority packets one-by-one.

In the Table 4, values of loss probabilities $P_{\text {loss }}^{(1)}, P_{\text {loss }}^{(2)}$, mean waiting time of type-2 packets $W_{1}^{(2)}$, second moment $W_{2}^{(2)}$ of type-2 packets waiting time distribution and jitter $J_{2}$

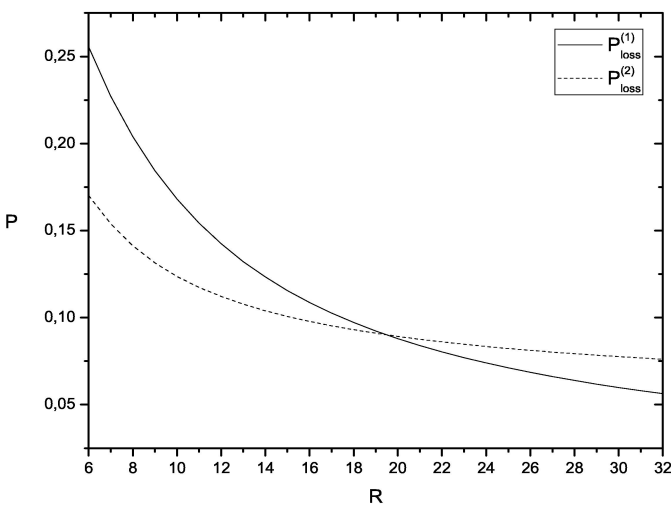

Fig. 6. Dependence of the loss probability $P_{\text {loss }}^{(1)}, P_{\text {loss }}^{(2)}$ on the total capacity $R$ of the buffer

as well as the analogous values $\widetilde{W}_{1}^{(2)}, \widetilde{W}_{2}^{(2)}, \widetilde{J}_{2}$ for waiting time distribution and jitter of type- 2 packets, which are not lost, the average number $L_{1}^{(1)}$ of type- 1 customers in the 
TABLE IV

Dependence of the Main Performance Measures on the Available Capacity $N$ of the Buffer for Priority Packets

\begin{tabular}{|c|c|c|c|c|c|c|c|c|c|c|}
\hline$N$ & $P_{\text {loss }}^{(1)}$ & $P_{\text {loss }}^{(2)}$ & $W_{1}^{(2)}$ & $W_{2}^{(2)}$ & $J_{2}$ & $\widetilde{W}_{1}^{(2)}$ & $\widetilde{W}_{2}^{(2)}$ & $\widetilde{J}_{2}$ & $L_{1}^{(1)}$ & $L_{1}^{(2)}$ \\
\hline 2 & 0.0118 & 0.3145 & 2.5817 & 28.9124 & 22.2474 & 3.7659 & 42.1750 & 27.9928 & 7.8928 & 0.6785 \\
\hline 3 & 0.0255 & 0.2065 & 3.6327 & 42.9386 & 29.7422 & 4.5783 & 54.1163 & 33.1551 & 10.5006 & 0.9284 \\
\hline 4 & 0.0393 & 0.1387 & 4.4055 & 54.8828 & 35.4747 & 5.1146 & 63.7175 & 37.5581 & 12.4283 & 1.1258 \\
\hline 5 & 0.0494 & 0.0995 & 5.0088 & 65.1451 & 40.0566 & 5.5621 & 72.3410 & 41.4039 & 13.6040 & 1.2627 \\
\hline 6 & 0.0563 & 0.0761 & 5.4357 & 73.3702 & 43.8235 & 5.8835 & 79.4152 & 44.7992 & 14.3147 & 1.3601 \\
\hline 7 & 0.0605 & 0.0626 & 5.7356 & 79.7129 & 46.8156 & 6.1186 & 85.0351 & 47.5982 & 14.7265 & 1.4257 \\
\hline 8 & 0.0631 & 0.0546 & 5.9391 & 84.4653 & 49.1922 & 6.2824 & 89.3477 & 49.8789 & 14.9665 & 1.4700 \\
\hline 9 & 0.0647 & 0.0500 & 6.0749 & 87.9102 & 51.0056 & 6.3949 & 92.5408 & 51.6460 & 15.1044 & 1.4991 \\
\hline 10 & 0.0656 & 0.0474 & 6.1637 & 90.3538 & 52.3625 & 6.4701 & 94.8456 & 52.9831 & 15.1836 & 1.5180 \\
\hline 11 & 0.0661 & 0.0458 & 6.2208 & 92.0432 & 53.3446 & 6.5195 & 96.4626 & 53.9586 & 15.2285 & 1.5301 \\
\hline 12 & 0.0664 & 0.0449 & 6.2570 & 93.1891 & 54.0395 & 6.5513 & 97.5727 & 54.6533 & 15.2539 & 1.5377 \\
\hline 13 & 0.0666 & 0.0444 & 6.2795 & 93.9504 & 54.5182 & 6.5714 & 98.3176 & 55.1343 & 15.2680 & 1.5424 \\
\hline 14 & 0.0667 & 0.0441 & 6.2934 & 94.4475 & 54.8411 & 6.5839 & 98.8081 & 55.4600 & 15.2758 & 1.5453 \\
\hline 15 & 0.0668 & 0.0440 & 6.3018 & 94.7662 & 55.0540 & 6.5916 & 99.1247 & 55.6756 & 15.2800 & 1.5470 \\
\hline 16 & 0.0668 & 0.0439 & 6.3068 & 94.9672 & 55.1917 & 6.5962 & 99.3256 & 55.8155 & 15.2823 & 1.5481 \\
\hline 17 & 0.0668 & 0.0438 & 6.3097 & 95.0919 & 55.2790 & 6.5990 & 99.4507 & 55.9043 & 15.2835 & 1.5487 \\
\hline 18 & 0.0668 & 0.0438 & 6.3115 & 95.1678 & 55.3333 & 6.6006 & 99.5272 & 55.9596 & 15.2841 & 1.5490 \\
\hline 19 & 0.0668 & 0.0438 & 6.3124 & 95.2132 & 55.3663 & 6.6015 & 99.5732 & 55.9934 & 15.2844 & 1.5492 \\
\hline 20 & 0.0668 & 0.0438 & 6.3130 & 95.2399 & 55.3860 & 6.6020 & 99.6003 & 56.0136 & 15.2846 & 1.5493 \\
\hline 21 & 0.0668 & 0.0438 & 6.3133 & 95.2552 & 55.3975 & 6.6023 & 99.6159 & 56.0254 & 15.2846 & 1.5494 \\
\hline 22 & 0.0668 & 0.0438 & 6.3135 & 95.2638 & 55.4040 & 6.6025 & 99.6246 & 56.0321 & 15.2847 & 1.5494 \\
\hline 23 & 0.0668 & 0.0438 & 6.3135 & 95.2686 & 55.4077 & 6.6025 & 99.6294 & 56.0358 & 15.2847 & 1.5495 \\
\hline 24 & 0.0668 & 0.0438 & 6.3136 & 95.2711 & 55.4096 & 6.6026 & 99.6320 & 56.0378 & 15.2847 & 1.5495 \\
\hline 25 & 0.0668 & 0.0438 & 6.3136 & 95.2723 & 55.4106 & 6.6026 & 99.6333 & 56.0388 & 15.2847 & 1.5495 \\
\hline 26 & 0.0668 & 0.0438 & 6.3136 & 95.2730 & 55.4111 & 6.6026 & 99.6340 & 56.0393 & 15.2847 & 1.5495 \\
\hline 27 & 0.0668 & 0.0438 & 6.3136 & 95.2733 & 55.4113 & 6.6026 & 99.6343 & 56.0396 & 15.2847 & 1.5495 \\
\hline 28 & 0.0668 & 0.0438 & 6.3136 & 95.2734 & 55.4115 & 6.6026 & 99.6344 & 56.0397 & 15.2847 & 1.5495 \\
\hline 29 & 0.0668 & 0.0438 & 6.3136 & 95.2734 & 55.4115 & 6.6026 & 99.6345 & 56.0397 & 15.2847 & 1.5495 \\
\hline 30 & 0.0668 & 0.0438 & 6.3136 & 95.2735 & 55.4115 & 6.6026 & 99.6345 & 56.0397 & 15.2847 & 1.5495 \\
\hline 31 & 0.0668 & 0.0438 & 6.3136 & 95.2735 & 55.4115 & 6.6026 & 99.6345 & 56.0398 & 15.2847 & 1.5495 \\
\hline 32 & 0.0668 & 0.0438 & 6.3136 & 95.2734 & 55.4115 & 6.6026 & 99.6344 & 56.0397 & 15.2847 & 1.5495 \\
\hline
\end{tabular}

buffer, the average number $L_{1}^{(2)}$ of type-2 customers in the buffer, are presented along with the corresponding value of $N$.

Figures 8 and 9 present the dependence of the characteristics $P_{\text {loss }}^{(1)}, P_{\text {loss }}^{(2)}, \widetilde{J}_{2}$ on the buffer capacity $N$ available for the priority packets in the graphical form.

Experiment 5. The objective of the experiment is to find the buffer capacity $N$ for type-2 customers which minimizes the economic criterion $I_{C}$ called Weighted Grade of Service (WGoS). This criterion as assumed having the following form:

$$
I_{C}=\frac{\lambda_{1}}{\lambda_{1}+\lambda_{2}} c_{\text {loss }}^{(1)} P_{\text {loss }}^{(1)}+\frac{\lambda_{2}}{\lambda_{1}+\lambda_{2}}\left(c_{\text {loss }}^{(2)} P_{\text {loss }}^{(2)}+c_{W_{1}^{(2)}} W_{1}^{(2)}+c_{J_{2}} J_{2}\right)
$$

where $c_{W_{1}^{(2)}}$ is the penalty for the waiting of type-2 customer per unit of time, $c_{J_{2}}$ is the penalty for waiting time jitter of

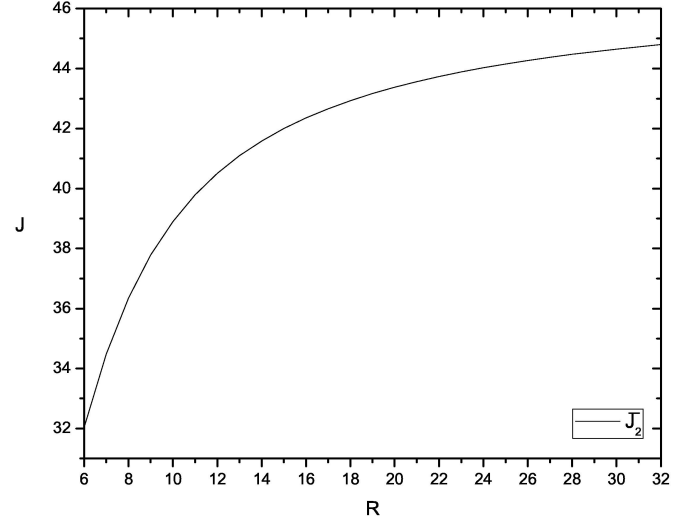

Fig. 7. Dependence of the jitter $\widetilde{J}_{2}$ of priority packets on the total capacity $R$ of the buffer 
type- 2 customer per the squared time unit; $c_{\text {loss }}^{(l)}, l=1,2$, is the penalty for a type- $l$ customer loss.

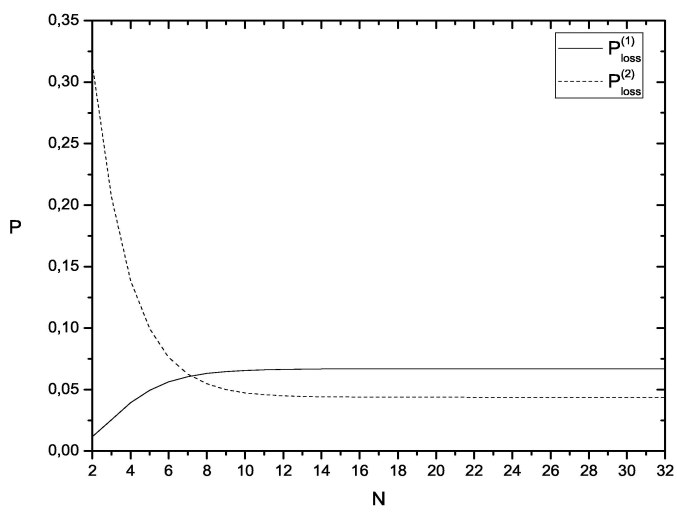

Fig. 8. Dependence of the loss probabilities $P_{\text {loss }}^{(1)}, P_{\text {loss }}^{(2)}$ on the available capacity $N$ of the buffer for priority packets

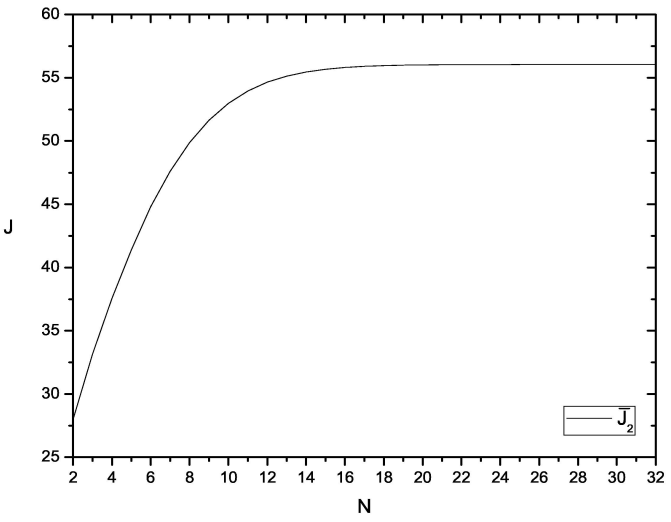

Fig. 9. Dependence of the jitter $\widetilde{J}_{2}$ on the available capacity $N$ of the buffer for priority packets

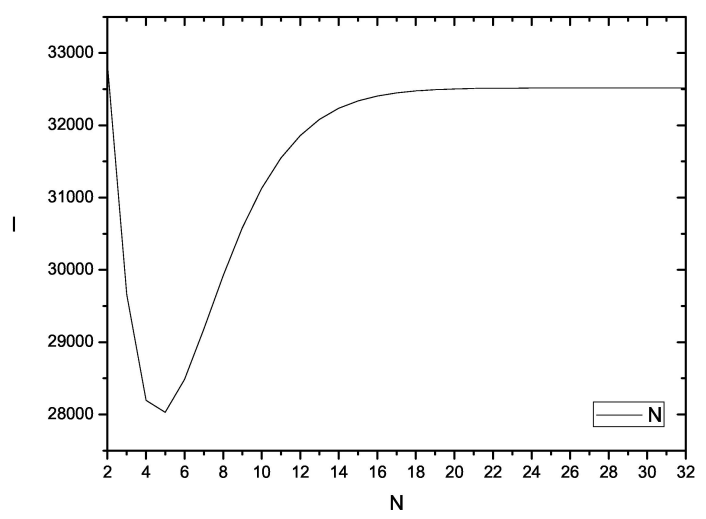

Fig. 10. Dependence of the economic criterion $I_{C}$ on the available capacity $N$ of the buffer for priority packets
In this experiment, the cost values are taken as follows:

$$
c_{W_{1}^{(2)}}=10, c_{J_{2}}=800, c_{\text {loss }}^{(1)}=100, c_{\text {loss }}^{(2)}=10^{5} \text {. }
$$

As it can be seen from Figure 10, the value of the WGoS criterion essentially depends on the buffer capacity $N$ and it is minimal when $N=5$.

\section{CONCLUSIONS}

The paper presents novel buffer management that combines both space and time priorities for different streams of the same multimedia traffic. The paper also presents a queueing model that captures some features of multimedia traffic that were not captured of any previous model in the literature including intra- and inter-stream correlation. The QoS measures obtained by the model allow real-time optimization of the buffer to fulfill the changing traffic requirements.

\section{REFERENCES}

[1] 3GPP TS 25.848. "Physical Layer Aspects of UTRA High Speed Downlink Packet Access", v4.0.0, Release 4. 2001.

[2] K. Al-Begain, I. Awan: Generalised Analysis of Buffer Management in Heterogeneous Multi-Service Mobile Networks, in Proc. of the UK Simulation Conference, March 2004, AlDabass, D. (Editor), St. Catherine College, Oxford, ISBN: 184233-099-3, 2004, pp. 87-93.

[3] A. Awan, K. Al-Begain: An Analytical Study of Quality of Service Provisioning for Multi-Service Mobile IP Networks Using Adaptive Buffer Management, in Proc. of the 11th International Conference on Analytical and Stochastic Modelling Techniques and Applications ASMTA04, June 2004, Al-Begain, K. and Bolch, G. (Editors), Magdeburg, Germany, ISBN: 3936150-38-9, 2004, pp. 166-172.

[4] T. Bonald, A. Proutiere: Wireless Downlink Data Channels: User Performance and Cell Dimensioning, in Proc. of Mobicom'03, San Diego, USA, 2003.

[5] S.R. Chakravarthy: The Batch Markovian Arrival Process: a Review and Future Work, in Proc. Advances in Probability Theory and Stochastic Process, Krishnamoorthy, A. et al. (Editors), NJ: Notable Publications, 2001, pp. 21-49.

[6] M.E. Crovella, A. Bestavros: "Explaining World Wide Web Traffic Self-similarity ", Boston University, 1995.

[7] A.N. Dudin, S. Chakravarthy: Optimal Hysteretic Control of the BMAP/G/1 System with Single and Group Service Modes, Annals of Operations Research, Vol.112, pp. 153-169, 2002.

[8] A.N. Dudin, G.V. Tsarenkov, V.I. Klimenok: Software "SIRIUS++" for Performance Evaluation of Modern Communication Networks, in Proc. of 6th European Simulation Multi-conference, Modelling and Simulation 2002, 1 Darmstadt, 2002, pp. 489-493.

[9] Q.-M. He: Queues with Marked Customers, Advances in Applied Probability, Vol.28, pp. 567-587, 1996.

[10] B.J. Kim, K.U. Hwang: Adaptive Bandwidth Allocation Method for Self-Similar Traffic, in Proc. of TMRC Workshop 2004 on Performance of Telecommunication Systems, Korea University, Seoul, 2004, pp. 21-34.

[11] V.I. Klimenok, A.N. Dudin: Application of Censored Markov Chains for Calculating the Stationary Distribution of the MultiDimensional Left-Skip-Free Markov Chains, in. Proc. of the International Conference Modern Mathematical Methods of Analysis and Optimization of Telecommunication Networks, Queues: flows, systems, networks, 23-25 September 2003, Gomel, 2003, Vol.17, pp. 121-128. 
[12] V.I. Klimenok, A.N. Dudin: Continuous Time Asymptotically Quasi-Toeplitz Markov Chains, in Proc. of the International Conference Mathematical Methods of Optimization Networks, Queues: Flows, systems, networks, 22-24 February 2005, Minsk, 2005, Vol.18, pp. 76-84.

[13] D.M. Lucantoni: New Results on the Single Server Queue with a Batch Markovian Arrival Process, Commun. Statist. - Stochastic Models, Vol.7, pp. 1-46, 1991.

[14] M.F. Neuts: "Matrix-Geometric Solutions in Stochastic Models", The Johns Hopkins University Press, Baltimore, 1981.

[15]H. van den Berg, R. Litjens, J. Laverman: HSDPA Flow Level Performance: The Impact of Key System and Traffic Aspects, in Proc. of COST290 TD(05)007, Colmar, 2005.

Khalid Al-Begain is Professor Mobile Networking in the School of Computing, University of Glamorgan. He obtained his BSc ('86), MSc ('88 with Distinction) and PhD degree ('89) in Communications Engineering from the Technical University of Budapest - Hungary. He has been an associate professor at the Department of Computer Science of Mu'tah University, Jordan. He then paid several research visits several universities in Italy. In 1997, he was awarded the Alexander von Humboldt Research Fellowship at the Department of Computer Science of the University of Erlangen-Nuremberg, Germany, where he established a research group on modelling of mobile networks and contributed to the invention of the Modelling, Specification and Evaluation Language - MOSEL. He also spent another year as guest professor as the Chair of Telecommunications at Dresden University of Technology supported by SEW-Eurodrive (Bruchsal-Germany) Grant where he led the research projects as part of a research project between the Chair and Lucent TechnologiesNuremberg. He has been an advisor for a modeling project for the research group of Lucent Technologies, Nuremberg/Germany. In 2000, he became the Director of research in the School of Computing of Bradford University in England before taking the chair at Glamorgan in 2003. Prof. Al-Begain is co-author the book "Practical Performance Modelling" published by Kluwer Academic Publishers in 2001 and more than 100 journal and conference papers. He has been the Guest Editor for several special issues of the international journals. He is UNESCO Expert in networking, UK Representative to EU COST Action 290 Management Committee on Traffic and QoS management of Wireless Multimedia Networks, Senior Member of the IEEE, Fellow and Charter IT Professional of the British Computer Society, Wales Representative to IEEE UKRI Computer Chapter Steering Committee and many other scientific organisations. Since 2003, he became the Conference Chair for the annual ASMTA (Analytical and Stochastic Modelling Techniques and Applications) Conference (ASMTA'03 in Nottingham, and ASMTA'04 in Magdeburg, Germany, ASMTA'05 in Riga, Latvia). He also the CoChair of PMW2MN05 held in Japan in July 2005. He will be the General Chair for the IEEE HSNMC'07 Conference to be hosted in Glamorgan.

Alexander N. Dudin has got Master degree in Applied Mathematics in 1976 from Belarusian State University, $\mathrm{PhD}$ - degree in 1982 from Vilnius University (now Lithuania) and Doctor of Science degree in 1992 from Tomsk University (now Russia). He had scientific visits to BEZ-Systems (USA), 1993, Free University of Amsterdam, 1995, Science University of Tokyo, 1997, Korean Advanced Institute of Science and Technology, 1998, Trier University, 2000, 2003, Glamorgan University (UK), 2005, Chonbuk National University (Korea), 2005. Currently he is the Head of the Laboratory of Applied Probabilistic Analysis in Belarusian State University, Professor of the Probability Theory and Mathematical Statistics Department. He is the Chairman of Organizing Committee of Belarusian Winter Workshops in Queueing Theory. Fields of scientific interests: stochastic processes, queues (controlled queues and queues in random environment, in particular) and their applications in telecommunications, databases, etc.

Vilena V. Mushko currently is the third year $\mathrm{PhD}$-student of the Department of Applied Mathematics and Computer Science in Belarusian State University. Her current field of scientific interests is queueing theory and its applications. 